\title{
Microstructure and Magnetic Properties of FePt/MgO Multilayers
}

\author{
Y. Fu*, J. E. Wittig*, J. Bentley**, N. D. Evans**, B. Lu*** \\ * Department of Electrical Engineering and Computer Science, Vanderbilt University, Nashville, \\ TN 37235 \\ ** Metals \& Ceramics Division, Oak Ridge National Laboratory, Oak Ridge, TN 37831 \\ *** Seagate Research, 1251 Waterfront Place, Pittsburgh, PA 15222
}

FePt is a promising candidate material for heat-assisted magnetic recording due to its large magnetocrystalline anisotropy [1]. However, to obtain such high anisotropy, as-sputtered films need to be annealed above $500^{\circ} \mathrm{C}$ to transform the disordered face centered cubic (FCC) phase to the ordered $\mathrm{L}_{0}$ phase [2]. Meanwhile, in order to facilitate perpendicular recording, $\mathrm{MgO}$ seedlayers and $\mathrm{MgO}$ laminations are used to promote FePt thin films grown along the [001] direction [3-5].

In this study, a series of $\mathrm{FePt} / \mathrm{MgO}$ multilayers were sputter deposited at room temperature as $\mathrm{Si} \backslash \mathrm{SiO}_{2}$ (substrate) $\backslash$ seedlayer(46 nm) $\backslash \mathrm{MgO} 6 \mathrm{~nm} \backslash[(\mathrm{FePt} X \mathrm{~nm}, \mathrm{X}=2.5,3,3.5,4) \backslash(\mathrm{MgO} 6 \mathrm{~nm})] \times \mathrm{x}$. The samples were characterized with a Philips CM20 in both the as-sputtered condition and after annealing at $700^{\circ} \mathrm{C}$ for 30 minutes. Selected-area diffraction (SAD) patterns indicated that the assputtered $\mathrm{FePt} / \mathrm{MgO}$ multilayers had strong (001) texture and post annealing successfully transformed the disordered FCC phase to the ordered L1 $1_{0}$ phase (Fig. 1). Plan view and crosssectional samples characterized by energy-filtered TEM (EFTEM) with a Philips CM30 equipped with a Gatan image filter (GIF) showed that Fe was not uniformly distributed between the $\mathrm{MgO}$ layers in the $X=2.5 \mathrm{~nm}$ sample after annealing, whereas continuous Fe layers were observed in the $X=4 \mathrm{~nm}$ sample even after annealing (Fig. 2). Spectrum lines acquired with a Philips CM200FEG operated in STEM mode and with Emispec Vision software revealed that the as-deposited FePt multilayers were not uniform in composition along the growth direction (Fig. 3a). However, this composition gradient was removed after annealing (Fig. 3b). For the magnetic properties, the coercivity was found to be inversely proportional to the thickness of the FePt layers (Table 1). This inverse proportionality might result from the discontinuity of the FePt between the $\mathrm{MgO}$ layers. For the thinner $\mathrm{FePt} / \mathrm{MgO}$ multilayers, the discontinuous FePt layer increases the coercivity since it behaves more like a granular medium where magnetic grains switch more coherently. On the other hand, the grains are more exchange coupled in the thicker $\mathrm{FePt} / \mathrm{MgO}$ multilayers due to the continuous magnetic layers and the switching mechanism is consequently less coherent [6].

References

[1] O.A. Ivanov, et al., Magat, Phys. Met. Metallog. 35, (1973) 81

[2] A. Cebollada, et al., Phys. Rev. B 50, (1994) 3419

[3] S, Jeong, et al., IEEE Trans. Magn. 36, (2000) 2336

[4] R. Mukai, et al., IEEE Trans. Magn. 39, (2003) 1925

[5] Z. G. Zhang, et al., Appl. Phys. Lett. 83, (2003) 1785

[6] This work was supported by Seagate Technology. Research at the ORNL SHaRE User Facility (JB, NDE) was supported by the Office of Basic Energy Sciences, U.S. Department of Energy under contract DE-AC05-00OR22725 with UT-Battelle, LLC. 

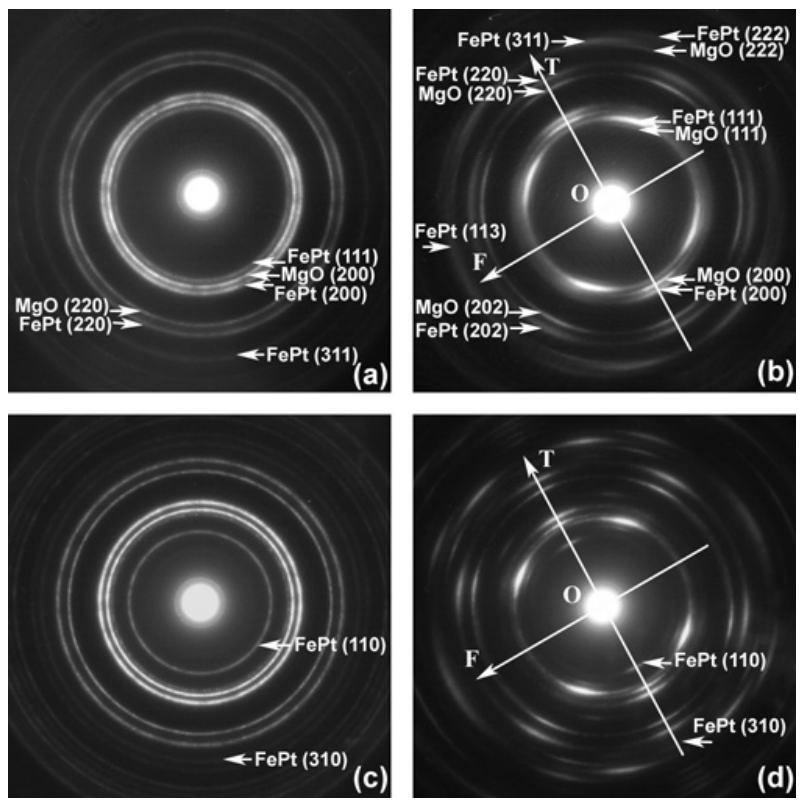

FIG. 1. SAD patterns of FePt/MgO

multilayer with $X=4 \mathrm{~nm}$. (a) $0^{\circ}$, (b) $60^{\circ}$ tilt in as-sputtered condition, (c) $0^{\circ}$, (d) $62^{\circ}$ tilt in annealed condition.

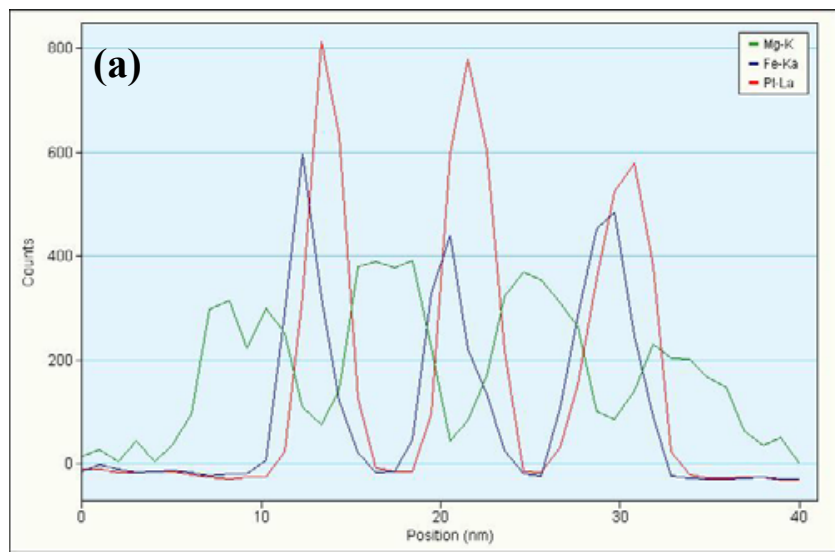

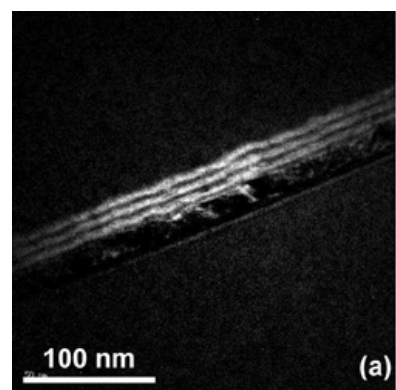
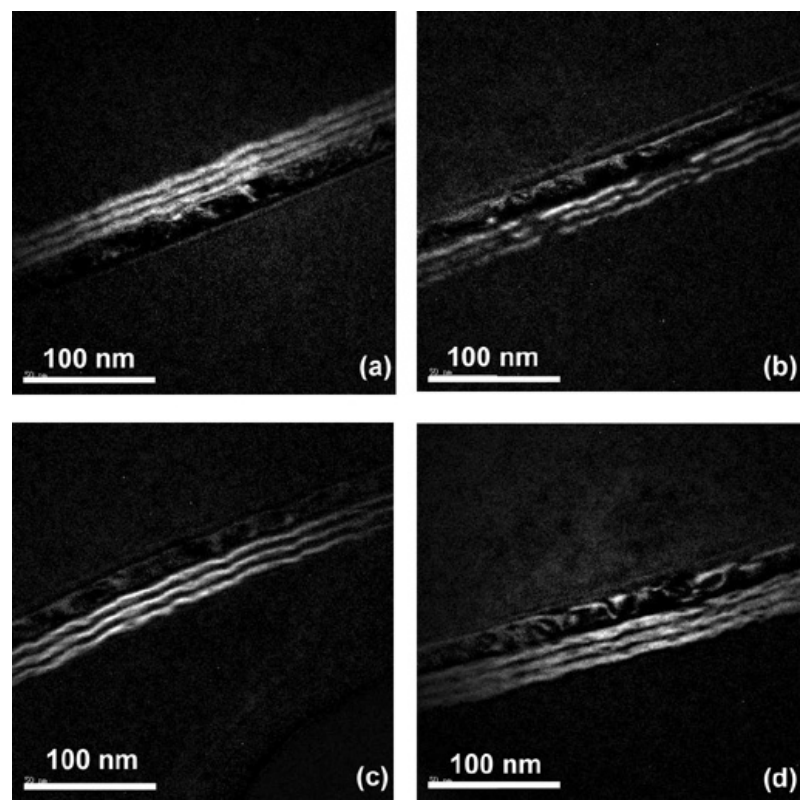

FIG. 2. EFTEM Fe maps of FePt $/ \mathrm{MgO}$ multilayers with $(a, b) X=2.5$ and $(c, d) X=4$ $\mathrm{nm}$ for $(\mathrm{a}, \mathrm{c})$ as-sputtered, and $(\mathrm{b}, \mathrm{d})$ annealed samples.

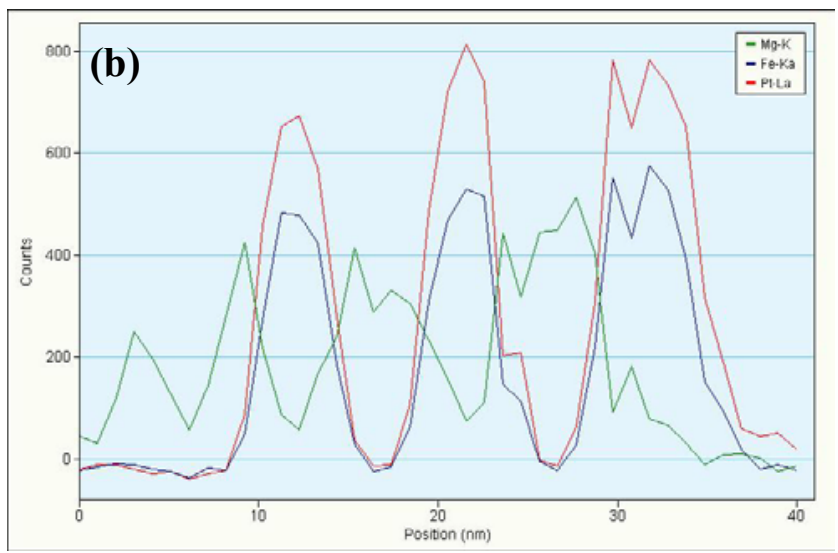

FIG. 3. Characteristic X-ray intensity profiles of the FePt/MgO multilayers with $\mathrm{X}=4 \mathrm{~nm}$. (a) as-sputtered, (b) after annealing.

TABLE 1. Coercivities and squareness ratios of annealed FePt/MgO multilayers

\begin{tabular}{lll}
\hline FePt layer thickness $(\mathrm{nm})$ & $\mathrm{H}_{\mathrm{c}}(\mathrm{kOe})$ & $\mathrm{S}$ \\
\hline 2.5 & 11.47 & 0.98 \\
3 & 10.58 & 0.99 \\
3.5 & 6.98 & 0.99 \\
4 & 6.51 & 0.99 \\
\hline
\end{tabular}

